\title{
Inferring Relations Between Color and Emotional Dimensions of a Web Site Using Bayesian Networks
}

\author{
Eleftherios Papachristos, Nikolaos Tselios, and Nikolaos Avouris \\ Human-Computer Interaction Group, Electrical and Computer Eng. Dept., \\ University of Patras, GR-265 00 Rio Patras, Greece \\ \{epap, nitse\}@ee.upatras.gr, avouris@upatras.gr
}

\begin{abstract}
In this paper, a novel methodology for selecting appropriate color scheme for a web site is presented. The methodology uses a machine learning algorithm for generating a network that relates the color model of a web site with the emotional values that are attributed to it by its users. The approach involves an empirical study to collect data, used to train the algorithm. A preliminary case study has been conducted to validate the applicability of the methodology. Description of the framework and of a set of tools that were built to support the methodology is also included.
\end{abstract}

\section{Introduction}

Various factors contribute to the effectiveness of a web site, such as structure, content hierarchy, styles, text format and navigational elements position. Additionally to these, one of the most important design factors that influence the usability of a site is effective color usage. Recent work has shown that the design space is affected by both engineering and aesthetic usability issues. Also, visual aesthetics of computer interfaces seem to be a strong determinant of users' satisfaction and pleasure [1]. The design has to communicate broader values such as sense of professionalism, skillfulness and credibility. Color plays various roles ranging from the aesthetic to the utilitarian. However, most prior studies and recent survey studies for design guidelines focus on cognitive efficiency evaluation of websites [2]. In addition, existing color guidelines are often of limited use. Experiential advice tends to be nonrepresentative, contradictory or even obsolete [3]. Even assuming that relevant guidelines exist, they are of little value to web designers with limited background on color theory and art. Furthermore, many issues remain unresolved, such the relationship between colors, the optimal number of colors for each case, etc.

The research goal described in this paper is to identify methodologically the color combinations which communicate effectively desired emotional values. For example, a news web site should effectively communicate values such as 'consistent', 'reliable', 'subjective', etc. Due to the fact that a direct evaluation of each color is heavily subjective, the methodology tackles this problem by indirectly evaluating the emotional influence of selected color combinations, and then breaking down the underlying influence of each factor via Bayesian Belief Networks. Tools have been developed to support the data collection and preprocessing stage, and to suggest appropriate chromatic combinations according to the desired communicating values. 
In the rest of the paper, the proposed methodology and the explicit research goals are presented first. Then, a case study, carried out to validate the effectiveness of the proposed framework, is described, together with the developed tools that support it.

\section{Color Scheme Selection Framework}

Our goal is to determine relations between color characteristics and affective, emotional descriptors. The result of this approach is a formal methodological process to select the appropriate color combination. In addition, for a web site of a given scope we can elicit the appropriate color characteristics. The proposed methodology is partially inspired from a similar research by Guerin et al. [4], who examined color usage in the frame of designing internal spaces. The subjects of the experiment had to evaluate computer generated interior environments with a list of 21 words, selected for this purpose, using a Likert scale from 1 to 5 .

In our study, a model of color has been developed, combining physical, aesthetic and artistic dimensions for various color combinations. A number of users (representing the expected users' characteristics) have been requested to characterize these color combinations according to a set of emotional descriptors. The 12 most distinctive terms were chosen: Pleasant, Formal, Fresh, Modern, Friendly, Aggressive, Professional, Attractive, Calming, Dynamic, Reliable and Sophisticated. These emotional dimensions are compatible with the thirteen emotional dimensions that have been used by Kim et al. [5]. This process led to a formal selection of chromatic combinations, in aspects such as dominant and secondary color selection, recommendation on number of colors to use, appropriate color scheme and secondary color scheme attributes such as degree of saturation and brightness. By simply asking the participants for their preferences in colors, their answers would present highly subjective character, thus leading to non meaningful or contradictory conclusions. Therefore, we constructed an indirect approach to gather their evaluations concerning the attributes of the color model of a specific layout. The data collected have been used for training a Bayesian belief network (BBN). A BBN is an annotated directed acyclic graph that encodes a joint probability distribution and is considered as a significant knowledge representation and reasoning tool, under conditions of uncertainty [6], [7]. The proposed framework is supported by two tools. The first to facilitate user data collection. The second tool allows the user to choose which emotional descriptor has to be present on her web site and at what degree. According to user's emotional preferences, the system proposes a list of color characteristics coupled with detailed explanations on the results.

\section{Validation of the Proposed Framework}

An experiment took place with the objective to validate the feasibility of the proposed framework. Forty six (46) participants (35 male and 11 female), aged 21 to 31, most of them experienced Internet users, evaluated eight color schemes each. Most participants were students of our Department. All of the characteristics referring to color selections were encoded to uniquely identify each layout. These comprise a 
color model that contains attributes related to the physical properties of the primary and secondary color (e.g. color hue, saturation and brightness), as well as aesthetic properties (e.g. perceived warmth of the primary color, color scheme). The data collected was 4416 evaluations ( $=46$ participants $x 12$ descriptors $x 8$ layouts).

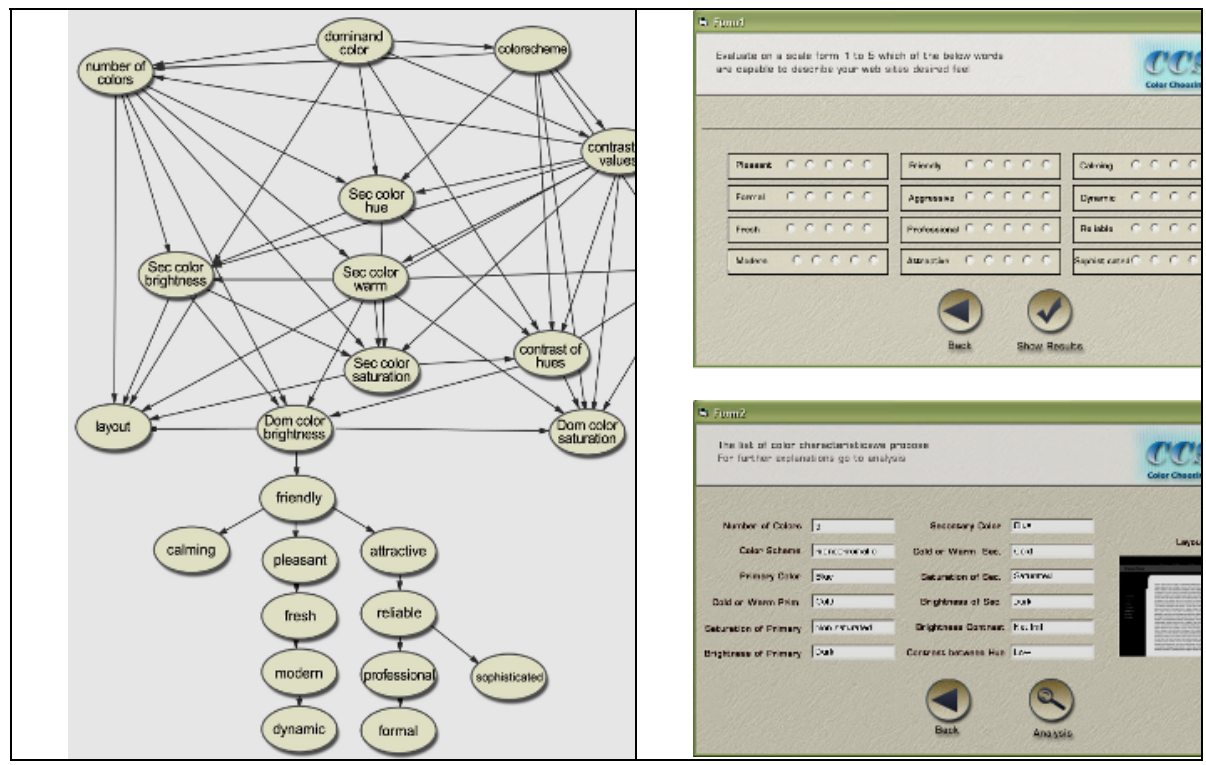

Fig. 1. The Bayesian Belief Network derived (left). Color Selector Tool (right). (a) the user has to select the desired emotional values. (b), the system infers the appropriate color characteristics using the trained Bayesian Belief Network

The result of the experiment is expressed through the Bayesian Belief Network constructed (Figure 1). With the help of the BBN construction and testing tool, Hugin Expert, the Bayesian Network built was analyzed. The diagrammatic nature of the BBN, allows recognition of the relations between the variables of the experiment (color scheme attributes and emotional descriptors). Furthermore, the method allows us to gain an insight on how a change in the value of one variable affects the attributes of other variables of the Network. In general, conclusions from the BBN are both structural and statistical. For example, according to the tree structure, the brightness of the dominant color has a stronger emotional effect than its hue.

Further analyzing the BBN structure, lead to a categorization of influence of color factors, into 4 levels of importance. The design attribute with the strongest effect is the brightness of the dominant color. Secondly, the brightness level of the secondary color and its type (warm or cold), the number of colors, contrast between hues. Thirdly, the two primary colors, the color scheme and the contrast level. Finally the dominant color's saturation and its type (warm or cold).

Besides observations on the structure of the network, or performing sensitivity studies on various nodes, other significant relationships have been derived. For example, it seems to be preferable to use more than four colors or monochromatic and 
analogical chromatic combinations. Additionally to this, it seems to be preferable to use low contrast levels between colors. Cold colors are perceived as more suitable than warm ones. And, if we would like to adopt a monochromatic color scheme, it is preferable to have high contrast in brightness. Finally, regarding saturation, it was found that the best combination is high saturated dominant color with low saturated secondary color.

\section{Conclusions}

In this paper, a novel framework has been presented that relates the web site color scheme to its emotional impact. It generates a network that relates the color model of a web site with the emotional values that are attributed to it by its users. We consider the proposed framework to have some advantages compared to the most common approach, i.e. applying guidelines for color usage. The objective of the framework has been to derive conclusions, of general value and applicability. The preliminary case study reported here was conducted mainly to certify the applicability of the methodology. Many of the findings reported here confirm earlier empirical rules on color and current tendencies in web design, which is a significant indication of the validity of the proposed methodological framework.

An additional research goal is to compare our results with expert's views about best color usage in web design in order to establish the reliability of our approach. Finally, one may put the issue of appropriate chromatic combination to a broader context. We argue that various models of typical web user behavior, like information seeking, may be extended to take into account color issues since they influence our perception of the quality of the information environment and affect our interaction with it ${ }^{1}$.

\section{References}

1. Lavie, T. and Tractinsky, N., Assessing Dimensions of Perceived Visual Aesthetics of Web Sites, Int. J. of Human-Computer Studies, 60 (3):269-298, 2004

2. Wang, P., A survey of design guidelines for usability of web sites. Proceedings of the 2001 Human-Computer Interaction International Conference 1, 183-187, 2001.

3. Schwier, R.A. and Misanchuk, E.R., The art and science of color in multimedia screen design Proc. of Conf. of the Assoc. for Educ. Comm. and Technology, Anaheim, CA., 1995

4. Guerin, D. A., Park, Y., \& Yang, S., Development of an instrument to study the meaning of color in interior environments. Journal of Interior Design, 20 (2), 31-41, 1995.

5. Kim J., Lee J., Choi D., Designing emotionally evocative homepages: an empirical study of the quantitative relations between design factors and emotional dimensions, Int. J. HumanComputer Studies, 59, pp. 899-940, 2003.

6. Jensen F. V.. "Bayesian Networks and Decision Graphs". Springer. 2001.

7. Glymour C., Cooper G. (eds.). Computation, Causation \& Discovery. AAAI Press/The MIT Press, Menlo Park, 1999.

1 The reported research here is funded by the Hellenic Ministry of Education under the Pyhtagoras II-EPEAEK program, project:"Study and Development of models of information search in the web". 